\title{
Efficient Morphological Segmentation of Brain Hemorrhage Stroke Lesion Through MultiResUNet
}

\author{
R. Shijitha ${ }^{1, *}$, P. Karthigaikumar ${ }^{2}$ and A. Stanly Paul ${ }^{2}$ \\ ${ }^{1}$ Department of Biomedical Instrumentation Engineering, Avinashilingam Institute for Home Science and Higher \\ Education for Women, Coimbatore, India \\ ${ }^{2}$ Department of ECE, Karpagam College of Engineering, Coimbatore, India \\ ${ }^{*}$ Corresponding Author: R. Shijitha. Email: shijithar2@gmail.com \\ Received: 15 May 2021; Accepted: 02 July 2021
}

\begin{abstract}
Brain Hemorrhagic stroke is a serious malady that is caused by the drop in blood flow through the brain and causes the brain to malfunction. Precise segmentation of brain hemorrhage is crucial, so an enhanced segmentation is carried out in this research work. The brain image of various patients has taken using an MRI scanner by the utilization of T1, T2, and FLAIR sequence. This work aims to segment the Brain Hemorrhagic stroke using deep learning-based Multi-resolution UNet (multires UNet) through morphological operations. It is hard to precisely segment the brain lesions to extract the existing region of stroke. This crucial step is accomplished by this proposed MMU-Net methodology by precise segmentation of stroke lesions. The proposed method efficiently determines the hemorrhagic stroke with improved accuracy of 95\% compared with the existing segmentation techniques such as U-net++, ResNet, Multires UNET and 3D-ResU-Net and also provides improved performance of $2 \mathrm{D}$ and 3D U-Net with an enhanced outcome. The performance measure of the proposed methodology acquires an improved accuracy, precision ratio, sensitivity, and specificity rate of $0.07 \%$, $0.04 \%, 0.04 \%$, and $0.05 \%$ in comparison to U-net, ResNet, Multires UNET and 3D-ResU-Net techniques respectively.
\end{abstract}

Keywords: Brain hemorrhage; magnetic resonance imaging; segmentation; multi-resolutional U-Net; morphological operations

\section{Introduction}

Stroke is a crucial malady caused by irregular blood flow in a specific area of the brain which provokes damage to the brain cells and lacks delivering oxygen and nutrients to the brain tissues. As per the research, stroke is stated as a third vigorous disease with an increased death rate, so early detection and instantaneous stroke therapy can prevent deaths and mitigate disability by improving blood supply in the case of an ischemic [1] stroke or minimizing bleeding and lowering brain pressures throughout the case of a hemorrhagic stroke. The types of strokes are Ischemic stroke, Hemorrhagic stroke, Transient ischemic attack, Brain Stem Stroke, and Cryptogenic Stroke.

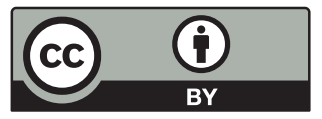

This work is licensed under a Creative Commons Attribution 4.0 International License, which permits unrestricted use, distribution, and reproduction in any medium, provided the original work is properly cited. 
Reference [2] Diverse imaging procedures (CT, MRI, carotid ultrasound, cerebral angiogram, and ECG) have been pursued to diagnose stroke. Stroke deprived of any symptoms becomes dangerous as it silently kills the brain cells. Segmentation of brain lesions is a critical role in stroke detection. Reference [3] It is an important measure in medical image processing. Also is used to extract various image attributes that could be utilized for image interpretation, classification, and comprehension. Image segmentation is used in medical imaging, form recognition, content-based image processing, robot vision, and other technology. Reference [4] Segmentation and thorough examination of lesions in medical images provide valuable evidence for neuropathology diagnosis and are critical for clinical planning, disease prevention, and patient outcome prediction. Quantitative imaging may reveal information about disease characteristics and their impact on complex anatomical structures, enabling research to gain knowledge about disease pathophysiology. The relationships [5] between different lesion types, their spatial distribution, and extent, as well as acute and chronic effects, are still frequently overlooked. Reference [6] Morphological image processing adopts the aim of eliminating these defects by optimizing the shape and composition of the image. In recent decades, several lesional stroke segmentation approaches have been proposed, which have been classified as supervised [7] or unsupervised depending on whether prior knowledge is needed. Unsupervised approaches do not require prior marking information and instead of analyzing data based on similarities. Matching filtering, morphological sorting, vessel monitoring, multi-scale [8] analysis, and model-based algorithms are some of the methods in these approaches. Normalized segmentation results in the lesional stroke detection with limited accuracy, but the morphological segmentation results from the morphological operation in the feature of the brain lesions results with improved accuracy. Considering the primary goal of the stroke segmentation through multiple input U-Net brain parcellation [9] that aims for better segmentation [10] in the baseline channel. Also provides improved performance of 2D and 3D U-Net with an enhanced outcome. But a large proportion of the computation time, which may become a possible constraint of this work. To improve the proposed pipeline's segmentation precision for small lesions is still a difficult challenge. Moreover, significantly boost the computational performance of the proposed pipeline. Furthermore, iterating the segmentation procedure so that the lesion areas found in the current iteration can be used as an external feedback source in the next iteration is one possible solution. Patch wise [11]. An evaluation of UNet++ over U-Net and broad U-Net designs in a variety of medical picture segmentation tasks has been carried out in earlier research. However the technique still has to be improved in terms of accuracy [12]. A multi-resolution UNet-based method has been used to increase the performance of medical picture segmentation. However, the optimum collection of neural network also for system must be performed more thoroughly. Reference [13] segmentation using fully convolutional pixel-labeling networks (ResNet) has been employed in which the segmentation accuracy has to be enhanced. Reference [14] 3D deep neural network for semantic segmentation of hemorrhage lesions has been evaluated in earlier approaches but lacks proper complete scanning is due to its downgraded efficiency. Fig. 1 depicts the normalized and morphological segmentation strategy and Fig. 1 depicts the normalized and morphological segmentation strategy.

This paper proposes a morphological segmentation through the multiresolution UNet method to modify the representation of an image into something more meaningful and easier to analyze any type of medical image. Segmentation involves separating an image into regions corresponding to objects. The goal of image segmentation is to cluster pixels into salient image regions, i.e., regions corresponding to individual surfaces, objects, or natural parts of objects. This approach was extended to the complete segmentation method by using the morphological operation. The not yet segmented image region is taken as a seed point. After segmentation, the infected region 
is identified by comparing the values of the original image with the values of the reference image. This enables a precise segmentation of brain hemorrhage stroke. Section 2 progress with the review of the existing methodologies, their measures, and precise problem statements present in each methodology has efficiently emphasized.

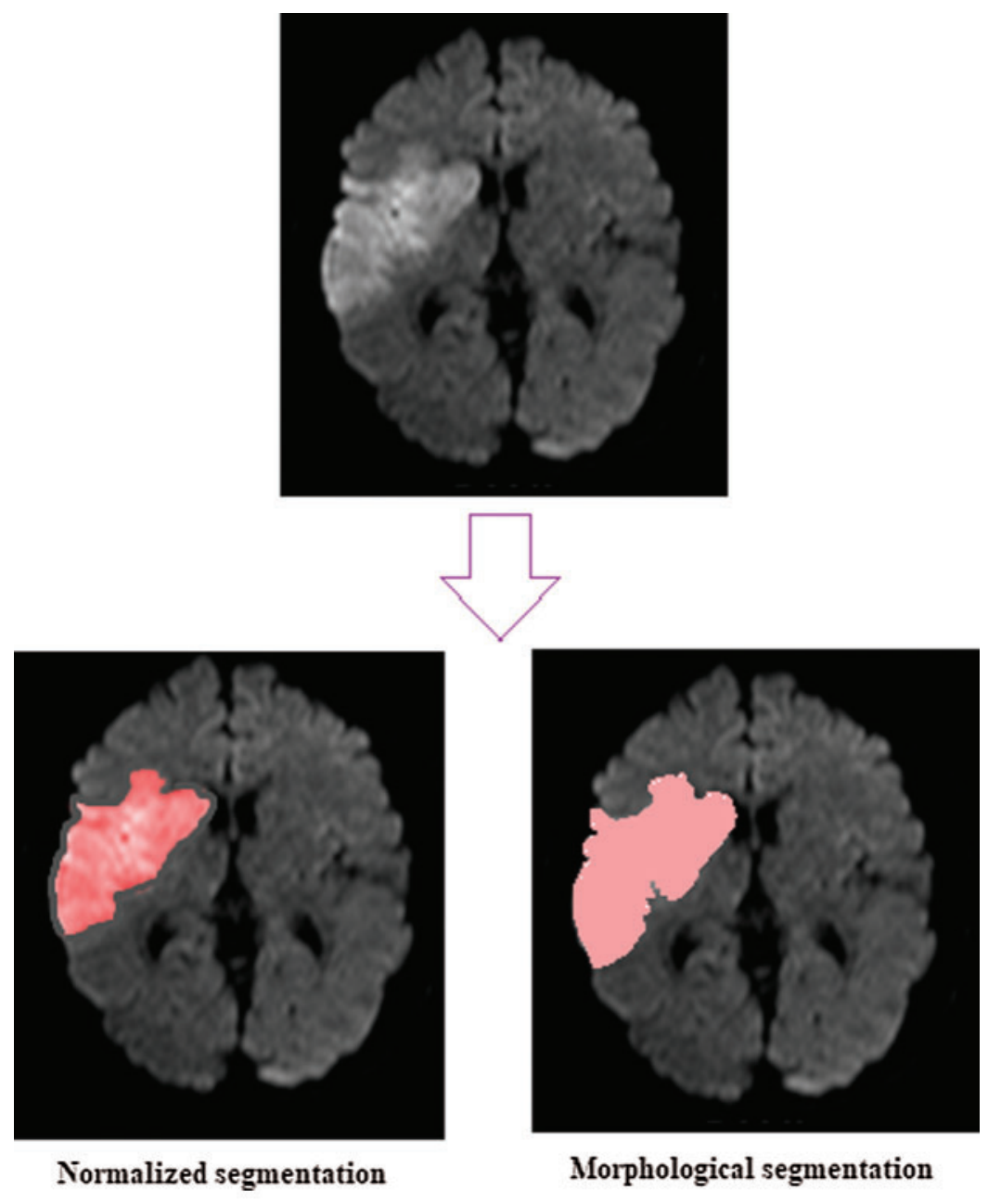

Figure 1: Normalized vs. morphological segmentation

\section{Literature Survey}

A unified cGAN (conditional Generative Adversarial Network) with data augmentation context has been established by $\mathrm{Wu}$ et al. [15]. A controlled segmentation through CNN (Convolutional Neural Network) has been undertaken for the lesional segmentation strategy. The brain MRI's extracted from the lesion mask from the adoptive cGAN enables dataset augmentation to progress the training process along with CNN using FSM (feature similarity module). The defect of this methodology is indicated as to enhance the authenticity and to clarify the assortment present in the brain MRI by upgrading the quantitative and qualitative analysis of the MRI. 
An automatic medical image segmentation strategy based on the deep learning technique has been established by Müller et al. [16]. CNN has been employed to enable medical image segmentation. Also, the proposed method contributes to developing a quicker construction of medical image segmentation through an application program. The accomplishment of segmentation with a limited line of code has been applied in this work. But the research mainly lacks data augmentation and processing of image and efficiency has to be improved.

A multiscale deep learning (DL) technique for the efficient segmentation of the brain lesion has been implemented by Konstantinos Kamnitsas et al. [17]. The proposed methodology is comprised of a capable and solid dataset for enhancing the processing step of 3D images. Furthermore, the presence of a dual pathway network enables the processing of input datasets into multiple scales instantaneously. The drawback involves a generative model for the data collection process to be improved and using it in the data augmentation stage is one way to enable the CNN susceptible to data heterogeneity.

An automated feature extraction step for the precise segmentation of cerebral vasculature has been established by Aditi Deshpande et al. [18]. A Hessian-based probabilistic vessel-enhancing filtering has been utilized to enable the segmentation step by the abstraction of vascular features. Morphological evaluation to enable an automated strategy for segmentation has been carried out. The combination of proposed filtering with the active contour facilitates segmentation in both MRI and CT imaging. But the shortcoming of the work states that it must be further enhanced by the utilization of machine learning techniques.

A ResNet-based deep learning technique termed a learnable group convolution network for brain segmentation has been established by Liu et al. [19]. The proposed work compacts with minimizing the layer of convolution also with better segmentation. Also, the improve recognition capacity ensemble with the deep supervision by diminishing the overfitting present in them. But the limitation includes the necessity of employing the multiscale data for improved feature reclamation.

A multiple segmentation of brain hemorrhage using CT imaging has been established by Nugroho et al. [20]. The investigation over diverse methodologies particularly contour and water segmentation, Otsu methodology and so on using the value of HU (House-field unit) has been carried out. The area and the volume of the hemorrhage and the investigation over asymmetric blood flow either by the virtue of blockage or by the crack in blood vessels. The performance over the analysis of the bleeding area has to be enhanced in this work.

The subsequent Section 3 copes with the proposed framework comprising of feature extraction ensued with the morphological operation to spot out the area of hemorrhage stroke and the proposed step is terminated by the efficient segmentation through Multiresolutional UNET.

\section{Proposed Methodology}

For the segmentation of biomedical entities, the current approach involves numerous morphological operations. The brain is utilized for morphological stroke segmentation depending on the structuring elements. The comprehensive segmentation steps to achieve effective stroke segmentation are demonstrated in Fig. 2.

\subsection{Image Preprocessing}

The preprocessing step incorporates the progressing of the MRI data to withdraw the undesired antiquities and deformities present in the image, also intensifies the feature extracted data 
present in them. Skull-stripping is essential to the progress needed for the segmentation of the brain image. Due to the frequency response of the Gaussian filter, it is used as the smoothening filter for removing the tissues which are non-cerebral and for the elimination of unhealthy tissues. The main operation in the preprocessing step is skull stripping. In this step, the skull layer is removed along with the outer cortical layer to enhance the cortical lamination through skull stripping. Fig. 3 elucidates the skull-stripped hemorrhage brain stroke.

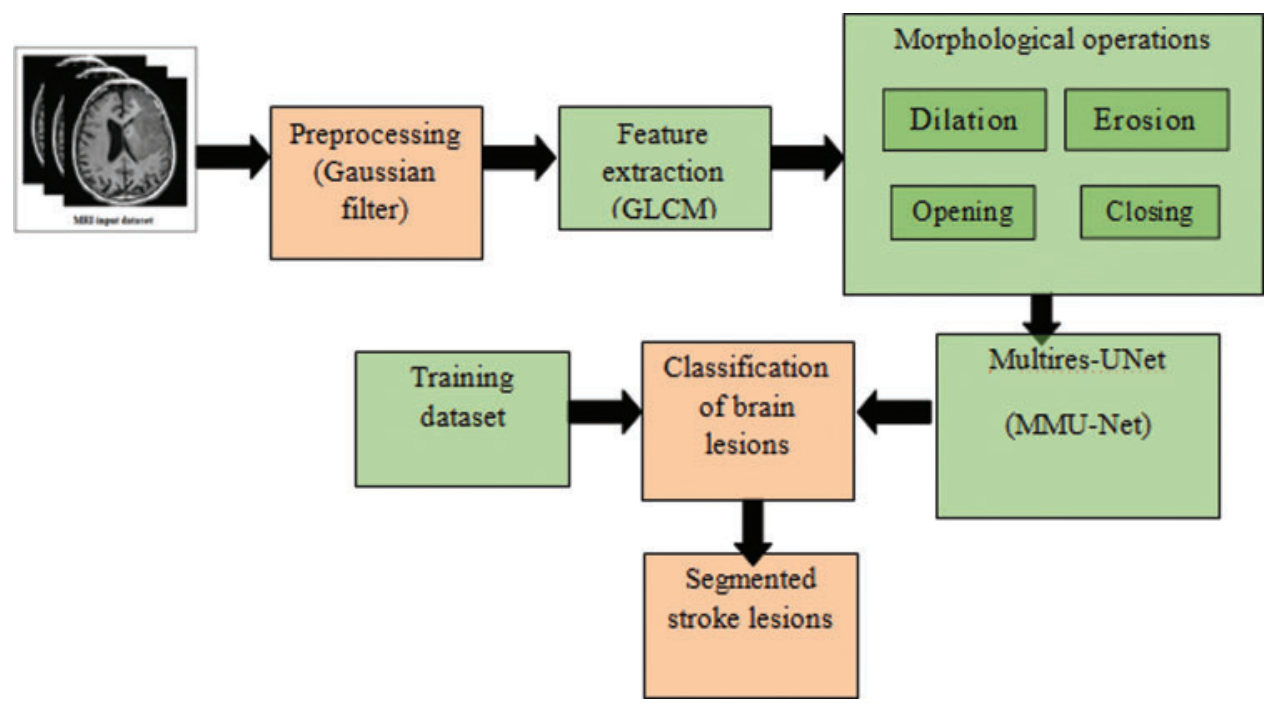

Figure 2: Overview of proposed block diagram for segmented brain stroke lesion

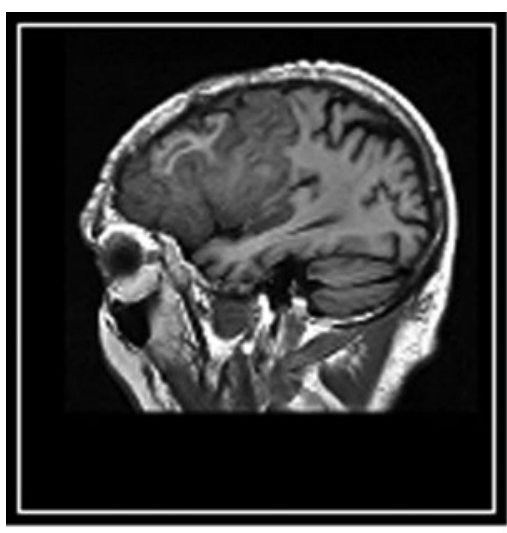

(a)

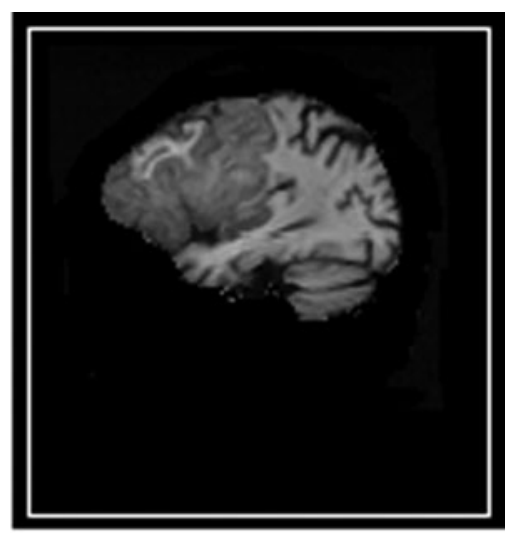

(b)

Figure 3: (a) Input image (b) Skull stripped image

\subsection{Feature Extraction}

Feature extraction is employed for better acknowledgment and classification of the images. It offers specific information about the image content and emphasizes certain properties of the image. In this work feature extraction is enabled by gray-level co-occurrence matrix The GLCM 
functions calculate how often pairs of pixels with given values and in defined spatial relations appear in an image, create a GLCM, and then extract quantitative measurements from this matrix to define the texture of an image. The segmentation for locating ROI isolates both in the ROI foreground and Centre region is done by extracting the feature of MRI which is shown in Fig. 4 below.

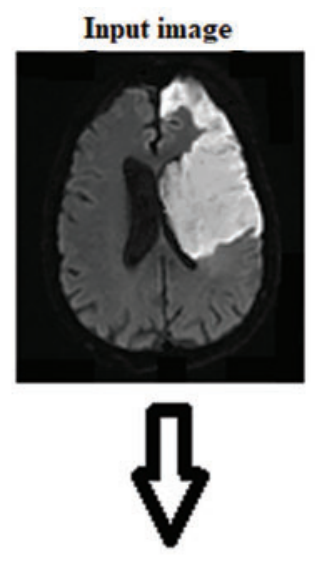

FLAIR

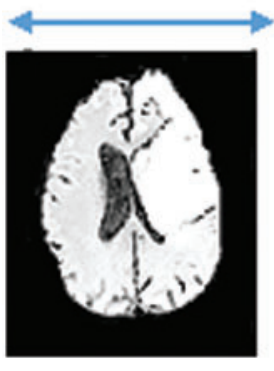

Tl

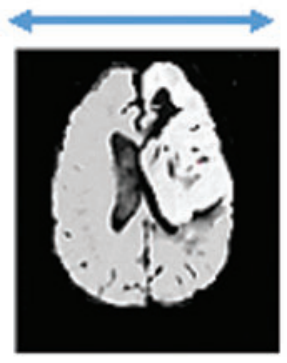

T2

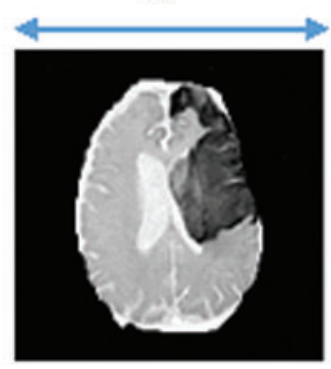

Figure 4: Feature extracted stroke image through GLCM

\subsection{Morphological MultiResU-Net Architecture}

The morphological MultiResU-Net with segmentation task has been evaluated in the subsequent step. Fig. 5 indicates the overall structure of the Morphological multi-resolution U-Net comprising of the morphological operation to obtain the efficient segmentation of the brain stroke lesions.

\subsubsection{Morphological Operations}

In this proposed methodology, four morphological operations were utilized for the segmentation strategy particularly Erosion, Dilation, Opening and closing. The objective of morphological image processing is to eliminate all of these flaws while retaining the image's structure. Only the related ordering of pixel values is acknowledged by morphological processes, not the pixel values among themselves. Usually is mainly oriented on binary pictures because of their numerical values, but it may also be used to grayscale pictures since their light transfer functions are uncertain and so their actual image pixels are ignored. Morphological techniques use a tiny template termed a structuring element to validate the image. This structuring element is adapted to all potential 
destinations in the source images and produces an outcome of a similar size. The edge pixels of the output pictures are based on comparable pixels in the input picture and their neighbors in this approach. This operation introduces a new binary image with a non-zero pixel value at that position in the input picture if the test result is found. Dilation, erosion, opening, and closing are the basic morphological operations, which are defined in logical AND, OR notation and defined by set analysis. Pixels are added via dilation, while pixels are removed by erosion at the object's borders. The structural element used to process the image determines whether pixels are removed or added.

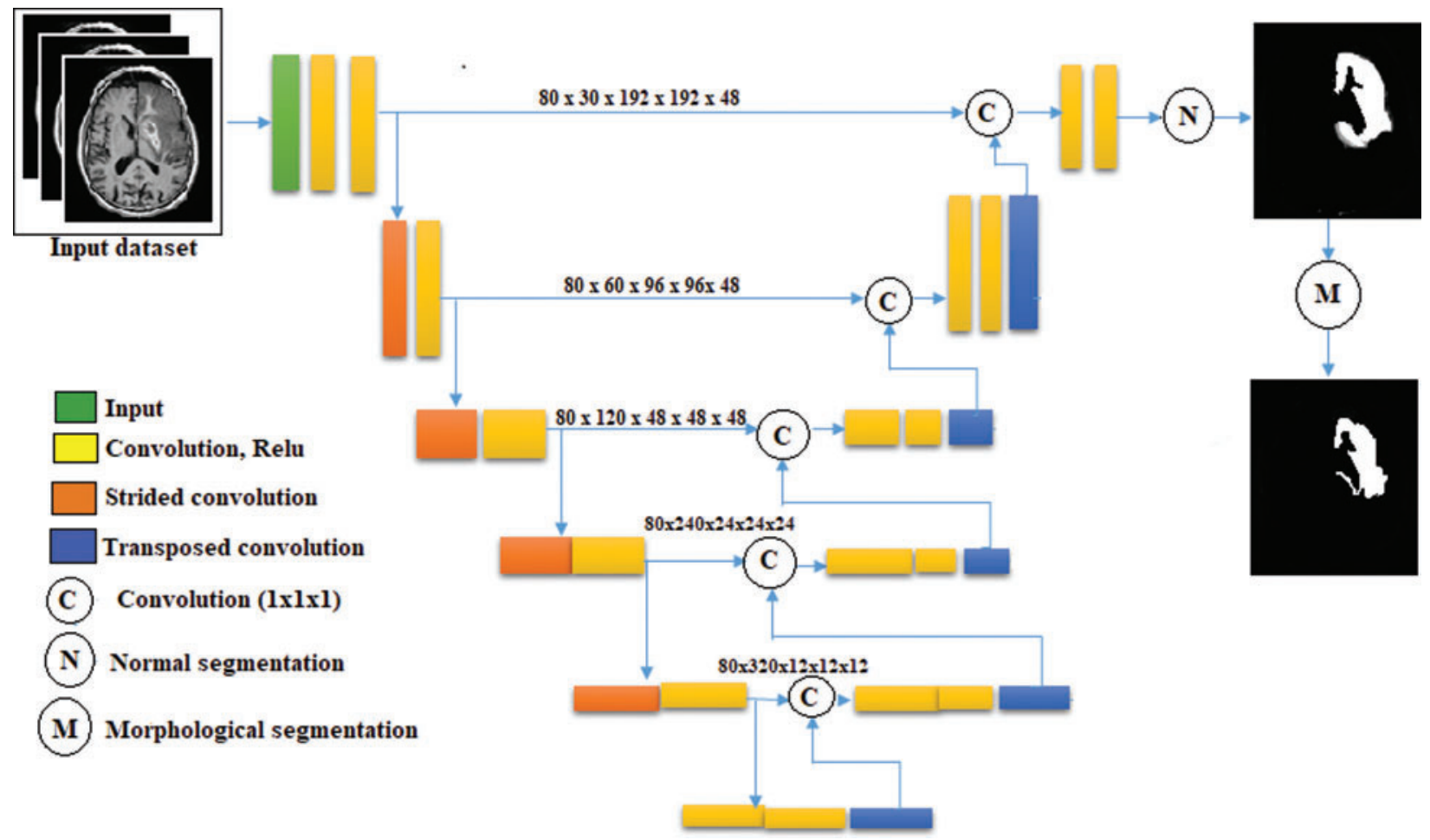

Figure 5: Morphological multiResU-Net architecture

To clean up the image, morphological approaches labeled as "opening-by-reconstruction" and "closing-by-reconstruction" are used. Flat maxima will be created inside each item that can be located as a result of these actions. Erosion is preceded by dilation in the opening, whereas opening-by-reconstruction is followed by morphological reconstruction. The black blotches and stem markings can be removed by shutting after the opening. A regular morphological closure is compared to a closure-by-reconstruction. Reconstruction-based opening and closing are more successful in erasing minor defects without changing the general forms of the objects than ordinary opening and closing.

\subsubsection{Morphological Operations}

In this proposed methodology, four morphological operations were utilized for the segmentation strategy particularly Erosion, Dilation, Opening and closing. The objective of morphological image processing is to eliminate all of these flaws while retaining the image's structure. Only the 
related ordering of pixel values is acknowledged by morphological processes, not the pixel values among themselves. Usually is mainly oriented on binary pictures because of their numerical values, but it may also be used to grayscale pictures since their light transfer functions are uncertain and so their actual image pixels are ignored. Morphological techniques use a tiny template termed a structuring element to validate the image. This structuring element is adapted to all potential destinations in the source images and produces an outcome of a similar size. The edge pixels of the output pictures are based on comparable pixels in the input picture and their neighbors in this approach. This operation introduces a new binary image with a non-zero pixel value at that position in the input picture if the test result is found. Dilation, erosion, opening, and closing are the basic morphological operations, which are defined in logical AND, OR notation and defined by set analysis. Pixels are added via dilation, while pixels are removed by erosion at the object's borders. The structural element used to process the image determines whether pixels are removed or added.

To clean up the image, morphological approaches labeled as "opening-by-reconstruction" and "closing-by-reconstruction" are used. Flat maxima will be created inside each item that can be located as a result of these actions. Erosion is preceded by dilation in the opening, whereas opening-by-reconstruction is followed by morphological reconstruction. The black blotches and stem markings can be removed by shutting after the opening. A regular morphological closure is compared to a closure-by-reconstruction. Reconstruction-based opening and closing are more successful in erasing minor defects without changing the general forms of the objects than ordinary opening and closing.

The sequence morphological operation includes the subsequent processing of erosion, dilation, opening and closing respectively. The detailed description of the processing is given in the following.

From the Fig. 6 the centre or occupied pixel is assigned to the maximum of its neighbors in dilation and to the lowest of its neighbors in erosion. Opening deals with the process of removing underlying artifacts from dilatation, whilst closing deals with process of closing perforations and smoothing the object shape by refilling in small gaps. Thus the morphological operation enables a crucial functioning for the segmentation step and the morphological strategy with multires UNet is given in the next section.

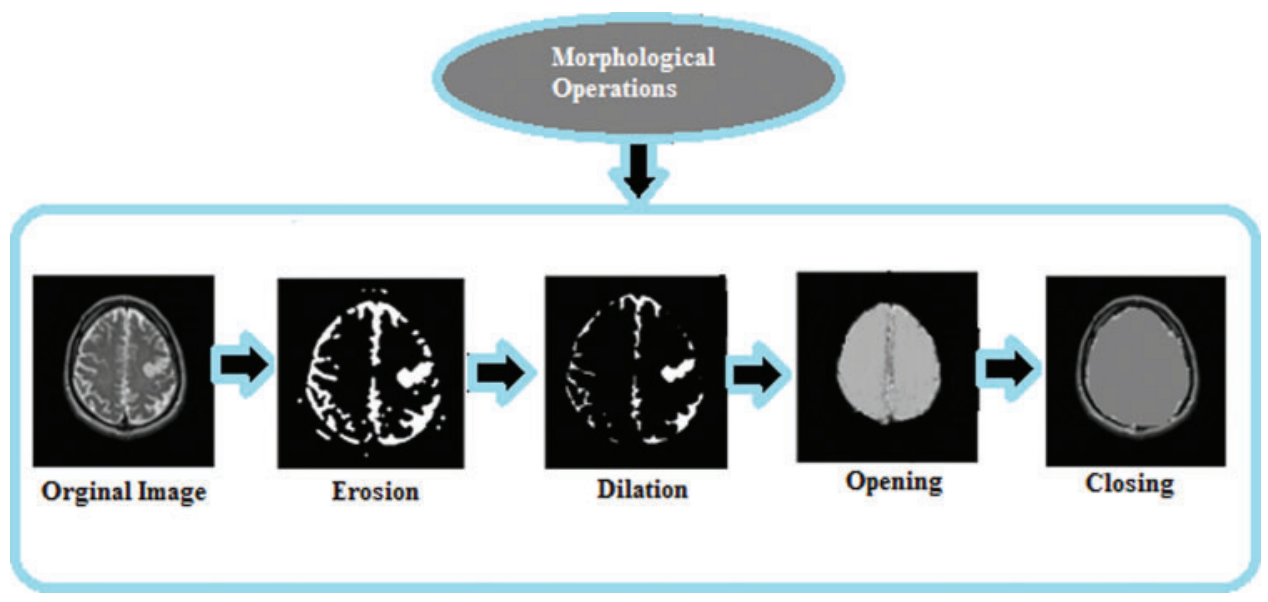

Figure 6: Morphological processing 
Assume that $\mathrm{K}$ is the binary image. The letter $\mathrm{S}$ stands for the structural element. $\mathrm{K} \Theta \mathrm{S}$ denotes the erosion of $\mathrm{K}$ by the structural element $\mathrm{S}$. It represents the set of all pixels that are contained within $\mathrm{K}$ when $\mathrm{S}$ is inserted at a certain pixel.

$K \Theta S=\{z /(S) z \subseteq I\}$

$\mathrm{K} \oplus \mathrm{S}$ is the dilation of $\mathrm{K}$ caused by the structural element $\mathrm{S}$. At least single element of $\mathrm{K}$ overlaps. Dilation is the process of enlarging a picture. It draws attention to tiny details in the image.

$K \oplus S=* z /() z \cap I \neq \phi\})$

The opening operation, symbolized by the letters I $\cdot \mathrm{S}$, smoothes an object's contour and splits the object's thin lines. The first step in the opening procedure is erosion, which is followed by dilation.

$K \cdot S=(F \Theta S) \oplus S$

Closing operation denoted by $\mathrm{K} \cdot \mathrm{S}$ smoothens the contours. It eliminates discontinuity and small gaps between the objects. In closing operation, dilation is performed followed by erosion.

$K \cdot S=(F \oplus S) \Theta S$

Thus the morphological operation enables a crucial functioning for the segmentation step and the morphological strategy with multires UNet is given in the next section.

\subsubsection{Multiresolution $U$-Net}

For accurate segmentation, U-Net uses a matrix of convolution layers similar to segNet and deep convolutional networks. An encoder and a decoder are used to extract unique image characteristics and create segmentation configurations, accordingly, in the device architecture. Convolution neural network with an order of $3 \times 3$ convolutions and max-pooling is simulated by the encoder. The max-pooling layer's order corresponds to two $2 \times 2$ layer progression signals. The recurrent method is repeated four times after downsampling, and the convolutional layer is advanced twice, linking the encoder and decoder. In contrast to the encoder, the decoder uses transposed convolutions with an order of $2 \times 2$ and a sequence of $3 \times 3$ convolution layers to upsample the data. The decoding phase uses similar processing as the encoder, with the mid-level filter. The certain convolution network, excluding the bottom layer, uses the Rectifier linear unit, and the bottom layer uses the sigmoid feature. The U-Net architecture's in Fig. 7 is the most groundbreaking feature was the implementation of skip connections. Before the encoder's pooling process, the output of the convolution layers is transferred to the decoder via all four levels.

The training of the dataset in the fully convolution layer with the dice of coefficient is given by,

The coefficient of dice is given by,

$C=\frac{2 T P}{2 T P+F P+F N}$ 


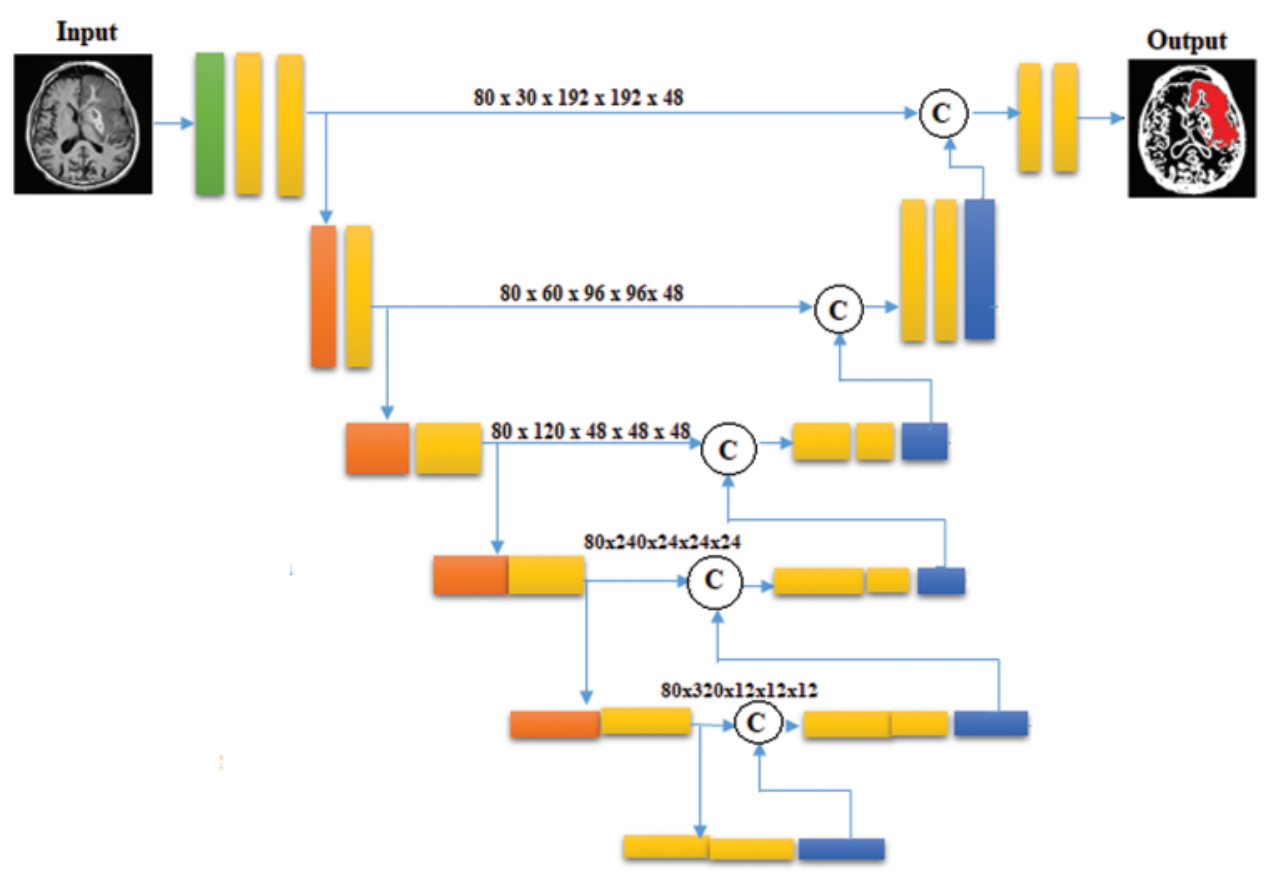

Figure 7: MultiResU-Net structure

In Eq. (1) $T P, F P$ and $F N$ represents the true positive, false positive and false negative voxels respectively.

$C=\frac{2 \sum_{u \in \Omega} A_{u} V_{u}}{2 \sum_{u \in \Omega} A_{u} V_{u}+\sum_{u \in \Omega}\left(A_{u}{ }^{2}-A_{u} V_{u}\right)+\sum_{u \in \Omega}\left(V_{u}{ }^{2}-A_{u} V_{u}\right)}$

The complete coefficient of dice can be written as

$$
\begin{aligned}
& C=\frac{2 \sum_{u \in \Omega} A_{u} V_{u}+Q}{\sum_{u \in \Omega} A_{u}^{2}+\sum_{u \in \Omega} V_{u}^{2}} \\
& \frac{\partial C}{A_{j}}=2\left[\frac{V_{j}\left(\sum_{u \in \Omega} A_{u}^{2}+\sum_{u \in \Omega} V_{u}^{2}\right)-2 A_{j}\left(\sum_{u \in \Omega} A_{u} V_{u}\right)}{\sum_{i \in \Omega} A_{u}^{2}+\sum_{u \in \Omega} V_{u}^{2}+Q}\right]
\end{aligned}
$$

From Eqs. (6)-(8) $A_{u}$ signifies anticipated binary segmentation, $V_{u}$ signifies ground truth binary volume and $Q$-indicates sample weighting. As compared to classical U-Net architecture, Multi-resolution U-Net is a prior art approach that replaces any encoder-decoder stage of architecture with multi-res blocks. It's made up of $3 \times 3$ convolution layers that are linked together to extract three-dimensional characteristics. A $1 \times 1$ convolution layer is added from the beginning to the end of the architecture to affix the information space and stabilize the communication. Shortcut connections, also known as residual paths (Res-Paths), are used to reduce differences in feature representation between encoder-decoder paired layers, which are made up of $3 \times 3$ and $1 \times 1$ convolution filters.

As the simple end-to-end network architecture in this analysis, a five-level U-Net is used in this methodology. To boost multi-scale image $\times$ function extraction, we tweaked the architecture. 
To remove some of the features directly from down-sampled iterations of the input image, certain convolutional layers to each stage of the contracting path (left side of the network) were added. To hold the output of the layers the same size as its input, we used zero-padding before each convolution layer.

The MultiResU-Net along with the morphological operation outweighs the Morphological MultiResU-Net. This methodology is used for the precise segmentation of the hemorrhagic lesion. The processing of the stroke lesion through the MMU-Net enables morphological segmentation through MultiResU-Net. Section 4 comprises the result and discussion of the proposed methodology.

\section{Result and Discussion}

The proposed methodology is implemented using the working platform of MATLAB 2017. The output of the MRI brain hemorrhage stroke segmentation methods and its performance are discussed and the comparison of different deep learning techniques is analyzed. The preprocessing of the hemorrhage lesions were carried out followed by the feature extraction. The morphological processing was consequently carried out with Multi-resolution UNET segmentation. The analyzed metrics are compared with the actual result and predicted result.

\subsection{Dataset Description}

The brain MRI is extracted using the BRAT 2018 scanner. T1, T2, and FLAIR are employed in an MRI scan to get a patient's brain image. The proposed Morphological Multi-resolution U-Net-based weighted MRI sample has been obtained within which the images were directed to the morphological operations precisely and also the segmented lesions were efficiently extracted out. In this proposed MRI Hemorrhage segmentation process. The brain image of various patients has taken using an MRI scanner. T1, T2 and FLAIR sequence was used for extracting the stroke lesions. The accurately segmented stroke lesions were abstracted from BRAT 2018 effectively.

The output of the MRI brain hemorrhage stroke segmentation methods which is shown in Fig. 8 and the output performance is measured by the subsequent measures of the morphological operations such as dilation, erosion, opening, closing and consequent function of Multi-resolution UNET segmentation.

\subsection{Performance Metrics}

The performance metric is esteemed based on the comparative measures of sensitivity, precision ratio, specificity, and sensitivity of the existing and proposed techniques. The metrics of 10 patients were evaluated by the following measures.

- Accuracy: Accuracy is defined as the proportion of perfectly classified illustrations of true positives and negatives. It's used to locate the odd pixel in an image. It's also used to examine to what extend an accurate segmentation process is done on an image. TP (true positive), TN (true negative), FP (false positive), and FN (false negative) measurements are used to assess the correctness of the proposed method.

- Precision ratio: Precision accurately defines the quality of certain irregularities in a given image. Precision, on the other hand, corresponds to the proportion of the results that is impactful. It emphasizes the result attributes that are more important.

- Sensitivity: Sensitivity is a highly accurate measure of a patient's fitness. It's used to explain a positive outcome correctly. The accuracy of the patients' predictions was determined by 
the compilation of test images. The study of high susceptibility results, aids in determining whether disease exists or not.

- Specificity: The ability to detect a patient's wrongly predicted disease is referred to as "specificity." It distinguishes between stable patients and those who are not. Specificity was used to extract the negative effects of the study. It decides the negative outcomes by recognizing true negatives in the picture.
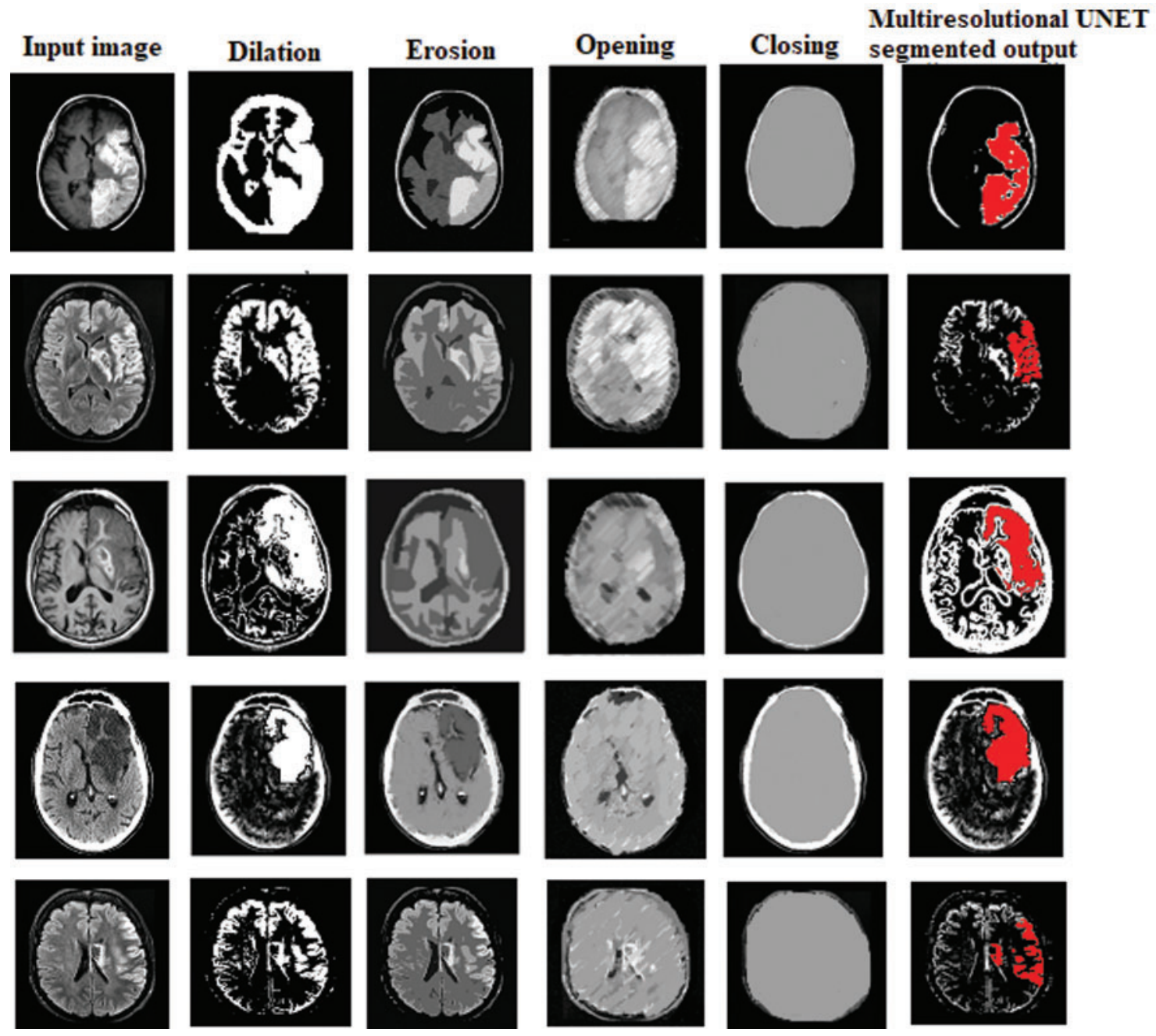

Figure 8: Segmented lesions of diverse patients through proposed methodology

The comparative analysis of U-Net, ResNet, Multires UNET and 3D Res U-Net by the measure of accuracy, precision rate, sensitivity and specificity is established in the following Tab. 1, represents the accuracy rate of the proposed technique where the accuracy is compared with various segmentation techniques such as U-net, Multires UNET, ResNet and 3D-ResUNet. The accuracy of the proposed method is $96 \%$, in which the accuracy of the existing techniques particularly U-Net, ResNet, Multires UNET and 3D Res U-Net is 74\%, 85\%, 89\%, $90 \%$ respectively. 
Table 1: Comparative analysis of accuracy with diverse segmentation techniques

\begin{tabular}{llllll}
\hline Patient & Accuracy & & & & \\
Fold No. & U-Net++ & ResNet & Multires UNET & 3D Res-U-Net & $\begin{array}{l}\text { Proposed morphological } \\
\text { multiResU-Net } \\
\text { (MMU-Net) }\end{array}$ \\
\cline { 2 - 5 } 1 & 0.72 & 0.86 & 0.82 & 0.89 & 0.97 \\
2 & 0.80 & 0.89 & 0.85 & 0.91 & 0.96 \\
3 & 0.69 & 0.88 & 0.81 & 0.9 & 0.98 \\
4 & 0.75 & 0.89 & 0.87 & 0.89 & 0.96 \\
5 & 0.82 & 0.90 & 0.83 & 0.9 & 0.92 \\
6 & 0.71 & 0.92 & 0.89 & 0.92 & 0.97 \\
7 & 0.73 & 0.91 & 0.87 & 0.93 & 0.96 \\
8 & 0.81 & 0.92 & 0.86 & 0.92 & 0.98 \\
9 & 0.74 & 0.88 & 0.84 & 0.91 & 0.95 \\
10 & 0.70 & 0.87 & 0.88 & 0.87 & 0.98 \\
Average & 0.74 & 0.89 & 0.85 & 0.90 & 0.96 \\
\hline
\end{tabular}

By comparing the proposed and the existing method, the accuracy of the proposed method has improved $0.07 \%$ from the existing method. The graphical depiction of the accuracy measure is given in Fig. 9.

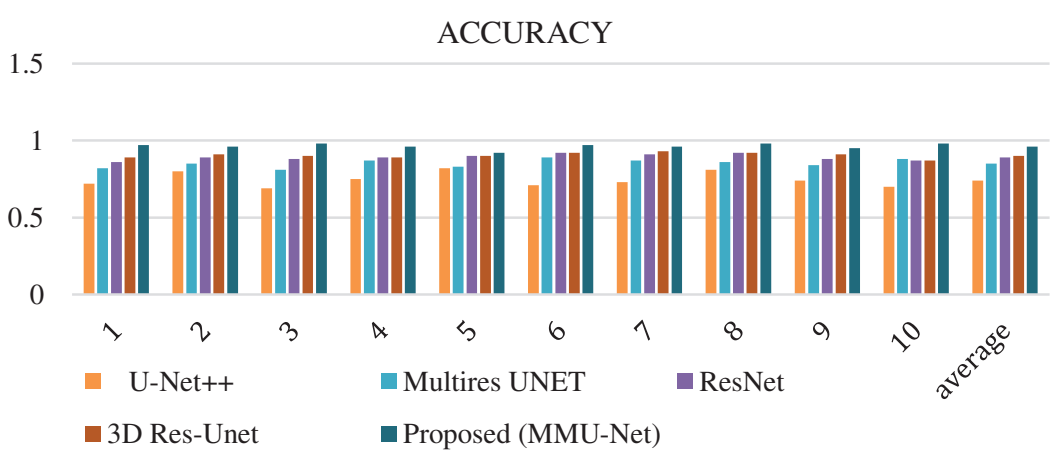

Figure 9: Performance metrics of accuracy

The performance metrics of precision ratio is given in Tab. 2 represents the precision rate of the proposed technique where the precision ratio is compared with various segmentation techniques such as U-net, ResNet, Multires UNET and 3D-ResU-Net. The precision ratio of the proposed method is $95 \%$, in which the precision ratio of the existing techniques particularly U-Net, ResNet, Multires UNET and 3D Res U-Net is $68 \%, 81 \%, 88 \%, 91 \%$ respectively.

By comparing the proposed and the existing method, the precision ratio of the proposed method has improved $0.04 \%$ from the existing method. The graphical depiction of the precision ratio measure is given in Fig. 10. 
Table 2: Comparative analysis of precision ratio with diverse segmentation techniques

\begin{tabular}{llllll}
\hline Patient & \multicolumn{2}{l}{ Precision ratio } & & & $\begin{array}{l}\text { Proposed morphological } \\
\text { multiResU-Net } \\
\text { Fold No. }\end{array}$ \\
\cline { 2 - 5 } & & & & & \\
\hline 1 & U-Net++ & ResNet & Multires UNET & 3D Res-U-Net & \\
2 & 0.68 & 0.82 & 0.73 & 0.93 & 0.95 \\
3 & 0.65 & 0.87 & 0.76 & 0.91 & 0.94 \\
4 & 0.61 & 0.77 & 0.68 & 0.88 & 0.92 \\
5 & 0.73 & 0.9 & 0.84 & 0.9 & 0.94 \\
6 & 0.67 & 0.89 & 0.78 & 0.91 & 0.93 \\
7 & 0.64 & 0.86 & 0.77 & 0.9 & 0.96 \\
8 & 0.75 & 0.91 & 0.86 & 0.93 & 0.98 \\
9 & 0.71 & 0.9 & 0.85 & 0.92 & 0.97 \\
10 & 0.67 & 0.92 & 0.89 & 0.94 & 0.96 \\
Average & 0.72 & 0.94 & 0.92 & 0.95 & 0.98 \\
\hline
\end{tabular}

PRECISION

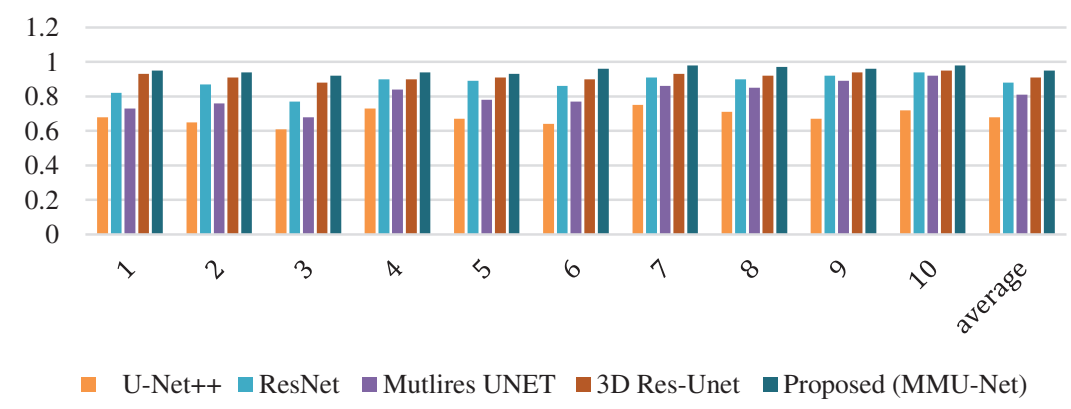

Figure 10: Performance metrics of precision ratio

The comparative analysis of sensitivity is given in Tab. 3, represents the sensitivity rate of the proposed technique where the sensitivity is compared with various segmentation techniques such as U-net, ResNet, Multires UNET and 3D-ResU-Net. The sensitivity of the proposed method is $94 \%$, in which the sensitivity of the existing techniques particularly U-Net, ResNet, Multires UNET and 3D Res U-Net is $68 \%, 86 \%, 88 \%, 90 \%$ respectively.

By comparing the proposed and the existing method, the sensitivity of the proposed method has improved $0.04 \%$ from the existing method. The graphical depiction of the sensitivity measure is given in Fig. 11.

The comparative analysis of specificity is given in Tab. 4, represents the specificity rate of the proposed technique where the specificity is compared with various segmentation techniques such as U-net, ResNet, Multires UNET and 3D-ResU-Net. The specificity of the proposed method is $94 \%$, in which the specificity of the existing techniques particularly U-Net++, ResNet, Multires UNET and 3D Res U-Net is $68 \%, 86 \%, 88 \%, 90 \%$ respectively. 
Table 3: Comparative analysis of sensitivity with diverse segmentation techniques

\begin{tabular}{llllll}
\hline Patient & Sensitivity & & & & \multicolumn{2}{c}{$\begin{array}{l}\text { Proposed morphological } \\
\text { multiResU-Net } \\
\text { Fold No. }\end{array}$} & & & & & U-Net++ \\
\cline { 2 - 5 } & ResNet & Multires UNET & 3D Res-U-Net & \\
\hline 1 & 0.65 & 0.90 & 0.87 & 0.92 & 0.95 \\
2 & 0.62 & 0.86 & 0.82 & 0.9 & 0.93 \\
3 & 0.72 & 0.88 & 0.85 & 0.89 & 0.89 \\
4 & 0.66 & 0.87 & 0.81 & 0.88 & 0.96 \\
5 & 0.73 & 0.85 & 0.87 & 0.86 & 0.9 \\
6 & 0.69 & 0.91 & 0.89 & 0.91 & 0.98 \\
7 & 0.80 & 0.89 & 0.87 & 0.9 & 0.96 \\
8 & 0.75 & 0.92 & 0.88 & 0.93 & 0.91 \\
9 & 0.60 & 0.87 & 0.86 & 0.88 & 0.94 \\
10 & 0.64 & 0.90 & 0.85 & 0.91 & 0.97 \\
Average & 0.68 & 0.88 & 0.86 & 0.9 & 0.94 \\
\hline
\end{tabular}

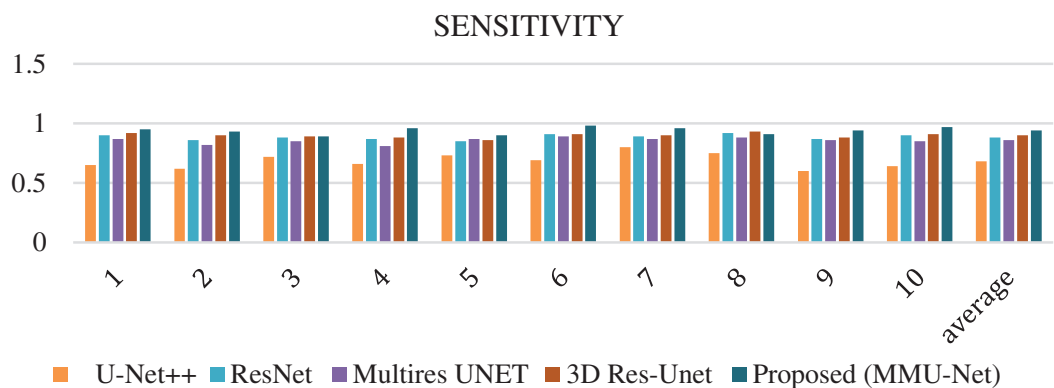

Figure 11: Performance metrics of sensitivity

Table 4: Comparative analysis of specificity with diverse segmentation techniques

\begin{tabular}{llllll}
\hline Patient & Specificity & & & & \multicolumn{2}{c}{$\begin{array}{l}\text { Proposed morphological } \\
\text { MultiResU-Net } \\
\text { Fold No. }\end{array}$} & & & & & \\
\cline { 2 - 5 } & & U-net++ & ResNet & Multires UNET & 3D Res-U-Net \\
1 & 0.69 & 0.84 & 0.78 & 0.92 & 0.96 \\
2 & 0.66 & 0.79 & 0.69 & 0.9 & 0.94 \\
3 & 0.72 & 0.82 & 0.76 & 0.89 & 0.92 \\
4 & 0.75 & 0.87 & 0.82 & 0.88 & 0.94 \\
5 & 0.67 & 0.9 & 0.89 & 0.92 & 0.95 \\
6 & 0.77 & 0.88 & 0.84 & 0.91 & 0.97 \\
7 & 0.81 & 0.89 & 0.86 & 0.9 & 0.93 \\
8 & 0.63 & 0.91 & 0.80 & 0.93 & 0.98 \\
9 & 0.60 & 0.83 & 0.73 & 0.89 & 0.94 \\
10 & 0.68 & 0.86 & 0.77 & 0.9 & 0.96 \\
Average & 0.70 & 0.86 & 0.79 & 0.90 & 0.95 \\
\hline
\end{tabular}


By comparing the proposed and the existing method, the specificity of the proposed method has improved $0.05 \%$ from the existing method. The graphical depiction of the specificity measure is given in Fig. 12.

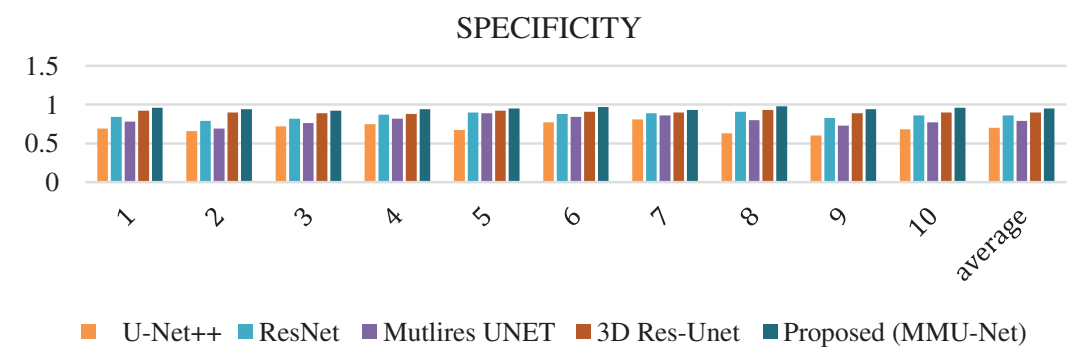

Figure 12: Performance metrics of specificity

\section{Conclusions}

A deep learning-based effective segmentation strategy of brain hemorrhage stroke using MMU-Net is carried out in this proposed work. Preprocessing step is used, to minimize noisy pixels in the database. The lesions are then segmented and enhanced using the morphological MultiRes U-Net. Different parameters such as accuracy, precision, sensitivity, and specificity are used to determine the quality of segmentation. To verify the progress of the proposed system, the output values of the MMU-Net are compared to the output values of other topologies such as U-net++, ResNet, Multires UNET and 3D-ResU-Net. According to the findings of the study, the suggested approach produces improved progression than existing techniques. Using morphological Muti-resolutional U-Net. The performance measures of the proposed scheme are correlated with the prior art techniques and acquired a performance improvement of 0.9 to $0.99 \%$.

\section{Future Works}

In the future, we plan to improve the image preprocessing by further optimization of our proposed system to achieve segmentation in a more precise manner, the attention mechanism will be added so that the extracted feature maps have different weights, which improves the utilization of feature information and more accurately locates the stroke.

Acknowledgement: The author with a deep sense of gratitude would thank the supervisor for his guidance and constant support rendered during this research.

Funding Statement: The authors received no specific funding for this study.

Conflicts of Interest: The authors declare that they have no conflicts of interest to report regarding the present study.

\section{References}

[1] J. Masci, J. Angulo and J. Schmidhuber, "A learning framework for morphological operators using counter-harmonic mean," in Proc. ISMM, Springer, Berlin, Heidelberg, pp. 329-340, 2013. 
[2] P. V. Ingole and K. D. Kulat, "A morphological segmentation based features for brain MRI retrieval," in Proc. IEEE Int. Conf. on Emerging Trends in Engineering \& Technology, Port Louis, Mauritius, pp. 210-214, 2011.

[3] N. Ironside, C. J. Chen, S. Mutasa, J. L. Sim, S. Marfatia et al., "Fully automated segmentation algorithm for hematoma volumetric analysis in spontaneous intracerebral hemorrhage," Stroke, vol. 50, no. 12, pp. 3416-3423, 2019.

[4] A. Sinha and J. Dolz, "Multi-scale self-guided attention for medical image segmentation," IEEE journal of biomedical and health informatics, vol. 25, no. 1, pp. 121-110, 2020.

[5] H. H. Chang and C. C. Hsieh, "Brain segmentation in MR images using a texture-based classifier associated with mathematical morphology," in Proc. EMBC, Jeju, Korea, pp. 3421-3424, 2017.

[6] D. Shen, G. Wu and H. I. Suk, "Deep learning in medical image analysis," Annual Review of Biomedical Engineering, vol. 19, no. 1, pp. 221-248, 2017.

[7] A. S. Lundervold and A. Lundervold, "An overview of deep learning in medical imaging focusing on MRI," Zeitschrift für Medizinische Physik, vol. 29, no. 2, pp. 102-127, 2019.

[8] S. H. Christy, "A novel approach for extricating information from speckle noise corrupted color brain MRI images using morphological enhancement followed by watershed and region growing based segmentation," in Proc. IEEE I2CT, Pune, India, pp. 1-4, 2018.

[9] D. Chudasama, T. Patel, S. Joshi and G. I. Prajapati, "Image segmentation using morphological operations," International Journal of Computer Applications, vol. 117, no. 18, pp. 16-19, 2015.

[10] C. U. P. Malla, M. D. C. Valdes Hernandez, M. F. Rachmadi and T. Komura, "Evaluation of enhanced learning techniques for segmenting ischaemic stroke lesions in brain magnetic resonance perfusion images using a convolutional neural network scheme," Frontiers in Neuro Informatics, vol. 13, pp. 33, 2019.

[11] R. Gu, G. Wang, T. Song, R. Huang and M. Aertsen, "CA-Net: Comprehensive attention convolutional neural networks for explainable medical image segmentation," IEEE Transactions on Medical Imaging, vol. 4, no. 2, pp. 699-711, 2020.

[12] N. Ibtehaz and M. S. Rahman, "MultiResUNet: Rethinking the U-Net architecture for multimodal biomedical image segmentation," Neural Networks, vol. 121, no. 11, pp. 74-87, 2020.

[13] Z. Zhou, M. M. R. Siddiquee, N. Tajbakhsh and J. Liang, "Unet++: A nested u-net architecture for medical image segmentation," in Deep Learning in Medical Image Analysis and Multimodal Learning for Clinical Decision Support. vol. 5. Cham: Springer, pp. 3-11, 2018.

[14] Y. Zhang, J. Wu, Y. Liu, Y. Chen, E. Wu et al., "MI-UNet: Multi-inputs UNet incorporating brain parcellation for stroke lesion segmentation from T1-weighted magnetic resonance images," IEEE Journal of Biomedical and Health Informatics, vol. 25, no. 2, pp. 526-535, 2020.

[15] W. Wu, Y. Lu, R. Mane and C. Guan, "Deep learning for neuroimaging segmentation with a novel data augmentation strategy," in Proc. 2020 IEEE EMBC, Montreal, QC, Canada, pp. 1516-1519, 2020.

[16] D. Müller and F. Kramer, "MIScnn: A framework for medical image segmentation with convolutional neural networks and deep learning," BMC Medical Imaging, vol. 21, no. 1, pp. 1-11, 2021.

[17] K. Kamnitsas, C. Ledig, V. F. Newcombe, J. P. Simpson, A. D. Kane et al., "Efficient multi-scale 3D CNN with fully connected CRF for accurate brain lesion segmentation," Medical Image Analysis, vol. 36, no. 5, pp. 61-78, 2017.

[18] A. Deshpande, N. Jamilpour, B. Jiang, P. Michel, Eskandari et al., "Automatic segmentation, feature extraction and comparison of healthy and stroke cerebral vasculature," Neuro Image: Clinical, vol. 30, pp. 102573, 2021.

[19] H. Liu, Q. Li and I. Wang, "A deep-learning model with learnable group convolution and deep supervision for brain tumor segmentation," Mathematical Problems in Engineering, vol. 3, pp. 61-78, 2021.

[20] K. Nugroho, T. A. Putranto, I. K. E. Purnama and M. H. Purnomo, "Multi segmentation method for hemorraghic detection," in Proc. IEEE ICoIAS, Singapore, pp. 62-66, 2018. 